\title{
Sparse Channel Estimation in OFDM Systems via Improved Tap Detection
}

\author{
Xiaolin Shi ${ }^{1,2}$, Yixin Yang ${ }^{1}$ and Long Yang ${ }^{1}$ \\ ${ }^{1}$ Northwestern Polytechnical University, Xi'an 710072, P. R. China \\ ${ }^{2} X i$ 'an University of Posts and Telecommunications, Xi'an 710121, P. R. China \\ linda20016@163.com,yxyang@nwpu.edu.cn,ylong311@126.com
}

\begin{abstract}
Many wireless channels encountered in practice tend to exhibit the structure of sparse multipath due to large bandwidth or large number of antennas. High-rate data wireless communications over multipath wireless channels usually require that the channel response be known at the receiver. In this paper, a novel scheme for the estimation of sparse wireless channels is developed. The initial estimation of the channel taps is obtained by the unstructured least-squares $(L S)$ method. Then, the presence of a channel tap is detected via an improved threshold obtained by applying the nature of sparse channels and the statistics of the noise vector. At last, the channel estimate is refined by deploying the knowledge previously acquired on the position of the nonzero taps and the structured LS method. The proposed method is compared and contrasted with the existing sparse estimation methods. And the results show the validity of the proposed method.
\end{abstract}

Keywords: Sparse channel estimation, Least-squares estimator, Tap detection, OFDM

\section{Introduction}

In a physical scattering environment, a radio signal emitted from a transmitter is reflected, diffracted, and scattered from the surrounding objects, and arrives at the receiver as a superposition of multiple copies of the transmitted signal, called multipath signal components. This multipath signal propagation leads to fading in the received signal strength that severely affects the rate and reliability of wireless communication systems [1]. However, if the knowledge of channel state information (CSI) is available at the receiver, it enables the exploitation of delay, Doppler, and/or spatial diversity to combat fading while further gains in rate and reliability are possible as well [1-2]. In practice, CSI is seldom available to communication systems a priori and the channel impulse response (CIR) need to be (periodically) estimated at the receiver in order to gain the reliable copy of the transmitted signals.

Physical arguments and growing experimental evidence suggest that many wireless channels encountered in practice tend to exhibit a sparse multipath structure at high signal space dimension in the sense that majority of CIRs being zero-value taps and a few nonzero taps[3-6]. Such sparse multipath communication channels include cellular communication environments, underwater acoustic channels, aeronautical communication channels, high-frequency radio communication channels and terrestrial high-definition television broadcasting channels. However, traditional training-based methods that rely on linear reconstruction schemes at the receiver seem incapable of exploiting the inherent low dimensionality of such sparse channels, thereby leading to overutilization of the limited communication resources of energy, latency, and bandwidth [2]. A number of researchers have tried to address this problem in the recent past and proposed training signals and reconstruction strategies that are tailored to the anticipated characteristics of sparse multipath channels [7-15]. 
Cotter and Rao proposed a sparse-channel estimation method based on the matching pursuit (MP) algorithm for frequency-selective single-antenna channels using singlecarrier waveforms [7-8]. By avoiding re-selecting the basis vectors, the orthogonal matching pursuit (OMP) algorithm has been shown to achieve much better performance [9]. However, the MP and OMP methods are very sensitive to the choice of the stopping rule. In contrast to the MP-based approach of [7-11], Raghavendra and Giridhar proposed a modified LS estimator in [12] for sparse frequency-selective single-antenna channels using multicarrier waveforms. The idea behind the approach of [12] was to reduce the signal space of the LS estimator by using a generalized Akaike information criterion to estimate the locations of nonzero channel taps. Finally, a somewhat similar idea was employed most recently in [13] for sparse frequency-selective single-antenna channels using single-carrier waveforms. In particular, one of the key differences between [12] and [13] is that [13] attempts to estimate the locations of nonzero channel taps by transforming the tap detection problem into an equivalent on-off keying detection problem.

The iterative detector/estimator (IDE) [13] offers improved performance with comparable complexity to MP without the need for channel order information. It achieves a mean-squared error (MSE) gain in channel estimation by applying parallel multiple thresholds for tap detections. The modified iterative detector/estimator (MIDE) algorithm [14] has improved performance than IDE by applying an iteration-varying threshold instead of multiple thresholds in parallel for each iteration in the IDE algorithm. However, the selection of the iteration-dependent threshold in MIDE lacks the theoretical articulation. In addition, a threshold-based approach [15] for sparse channel estimation in orthogonal frequency division multiplexing (OFDM) systems (named as TMSE) is derived by minimizing MSE per CIR coefficient and these thresholds are then applied on the CIR LS estimate to detect its structure. Unfortunately, MSE is very sensitive to tap missing and false detection that is likely to degrade the detection performance for channel taps.

Actually, if the nonzero tap locations have been properly detected, the estimation efforts are focused on a smaller number of parameters thus leading to a more accurate estimate of the channel coefficients. To achieve this goal, we propose sparse channel estimation with nonzero tap detection. In this paper, we have a three-stage scheme of the channel estimation. The first stage is an unstructured LS estimator, which yields detectable CIRs from the received signals. The second stage is a threshold-based tap detector, which determines the nonzero taps by using the statistics of noise and the nature of sparse channels. The final stage is a structured LS estimator, which precisely estimates the CIRs by exploiting the position knowledge of the nonzero taps obtained in the second stage.

The rest of this paper is organized as follows. The OFDM system model is described in Section 2. Section 3 introduces the proposed sparse CIR estimation method. In Section 4, the performance of the proposed method is verified through simulations. Section 5 draws the conclusion.

Throughout, vectors are represented as boldface lowercase letters and matrices as boldface uppercase letters. For vectors and matrices, $\mathbf{A}^{T}, \mathbf{A}^{H}, \mathbf{A}^{-1}$ and $\mathbf{A}^{+}$denote the transpose, the Hermitian transpose, the inverse and the pseudo-inverse of $\mathbf{A}$ respectively.

\section{OFDM System Model}

Without loss of generality, we consider an assisted OFDM system employing $N$ subcarriers with the parallel of $N_{d}$ data symbols and $N_{p}$ pilot symbols. The message sequence to be transmitted is mapped onto constellation points and modulated onto the data subcarriers. The pilot symbols are then multiplexed with data symbols. After OFDM modulation, $L_{c p}$ samples of cyclic prefix (CP) are added at the beginning of the $N$ samples 
of inverse discrete Fourier transform (IDFT) to form one OFDM symbol, which can be expressed as

$$
x_{n}=d_{n}+p_{n},-L c p \leq n \leq N-1 .
$$

Here, $d_{n}$ and $p_{n}$ represent the data and pilot symbols, respectively.

Since a variety of channels are prone to multipath propagation due to refraction, reflection, and scattering, the channel impulse response $h(k)$ has the form

$$
h(k)=\sum_{l=0}^{L-1} a_{l} \delta\left(k-\tau_{l}\right)
$$

where $\delta(\cdot)$ is the delta function. $a_{l}$ and $\tau_{l}$ is the amplitude and time-delay of the $l$ th path, respectively. $a_{l}$ is a zero-mean complex Gaussian random variable with $E\left[a_{i} a_{j}^{*}\right]=0$ and $E\left[\left|a_{l}\right|^{2}\right]=e^{-\beta l}$, where $\beta$ is the exponential power delay profile [12]. In the above model, path delays are sample spaced and $L$ denotes the delay spread of a channel, which is assumed to be approximately known at the receiver (e.g. via channel sounding techniques [16]). The OFDM symbols are then transmitted over a multipath fading channel. Assume the length of $\mathrm{CP}$ is larger than the maximum path delay to guarantee the orthogonality of subcarriers of the OFDM system, i.e., $L_{c p}>L$. To simplify analysis, we assume that synchronization is perfect at the receiver and the channel does not change during one OFDM symbol period.

After the CP is removed, the remaining samples are demodulated by the N-point discrete Fourier transform (DFT) operation. So, the received sample vector is expressed as

$$
\mathbf{y}=\mathbf{X F H}+\mathbf{n}_{f},
$$

where $\mathbf{y}=\left[y_{0}, y_{1}, \ldots, y_{N-1}\right]^{T}$ is the received symbols with the CP removed. $\mathbf{X}$ is the $N \times N$ diagonal matrix of transmitted data comprising data matrix $\mathbf{D}$ and pilot matrix $\mathbf{P}$, i.e., $\mathbf{X}=$ $\mathbf{D}+\mathbf{P}=\operatorname{diag}\left[x_{0}, x_{1}, \ldots, x_{N-1}\right]^{T} . \mathbf{F}$ is $N \times N$ DFT matrix and the channel vector $\mathbf{H}=[h(0), \ldots, h(L-1), 0, \ldots, 0]^{T}$. The zero-mean complex Gaussian noise vector $\mathbf{n}_{f}$ has its covariance matrix $\sigma_{f}^{2} \mathbf{I}_{N}$, i.e., $\mathbf{n}_{f} \sim C N\left(0, \sigma_{f}^{2} \mathbf{I}_{N}\right)$.

Generally, the received samples of pilot symbols are used for the channel estimation, which can be expressed as

$$
\mathbf{r}=\mathbf{P F} \mathbf{F}_{p} \mathbf{h} \mathbf{n}=\mathbf{S h}+\mathbf{n},
$$

where $\mathbf{r}=\left[r(0), r(1), \ldots, r\left(N_{p}-1\right)\right]^{T} . \mathbf{S}=\mathbf{P} \mathbf{F}_{p}$ is usually known as measurement matrix. $\mathbf{P}$ is the $N_{p} \times N_{p}$ diagonal matrix of the pilots. $\mathbf{F}_{p}$ is a $N_{p} \times L_{c p}$ matrix formed by the first $L_{c p}$ columns of the DFT matrix $\mathbf{F}$ in (3) and the $N_{p}$ rows of the selected matrix associated with the pilot subcarriers. The channel vector $\mathbf{h}=$ $\left[h(0), h(1), \ldots, h\left(L_{c p}-1\right)\right]^{T}$ and $\mathbf{n}$ is the additive white Gaussian noise vector.

\section{Proposed Sparse CIR Estimation Method}

The initial estimate of the sparse channel vector $\mathbf{h}$ can be obtained by the unstructured LS method:

$$
\hat{\mathbf{h}}_{L S}=\mathbf{S}^{+} \mathbf{r}=\left[\mathbf{S}^{H} \mathbf{S}\right]^{-1} \mathbf{S}^{H} \mathbf{r},
$$

where $\mathbf{S}^{+}=\left[\mathbf{S}^{H} \mathbf{S}\right]^{-1} \mathbf{S}^{H}$ is the pseudo-inverse of $\mathbf{S}$. Substituting (4) into (5), the above equation is expressed as

$$
\hat{\mathbf{h}}_{L S}=\mathbf{h}+\left[\mathbf{S}^{H} \mathbf{S}\right]^{-1} \mathbf{S}^{H} \mathbf{n} .
$$

We consider the case that the pilots are uniformly distributed and $\mathbf{S}^{H} \mathbf{S}=N_{p} \mathbf{I}$, the equation of (6) can be rewritten as: 


$$
\hat{\mathbf{h}}_{L S}=\mathbf{h}+\frac{1}{N_{p}} \mathbf{S}^{H} \mathbf{n}=\mathbf{h}+\mathbf{v} .
$$

where $\mathbf{v}=\left(1 / N_{p}\right) \mathbf{S}^{H} \mathbf{n}$ and it is still a zero-mean complex Gaussian vector with its covariance matrix $\mathbf{C}_{m}$ expressed as:

$$
\mathbf{C}_{m}=E\left(\mathbf{w}^{H}\right)=\frac{1}{N_{p}} \sigma_{f}^{2} \mathbf{I}=\sigma_{v}^{2} \mathbf{I} .
$$

where $\sigma_{v}^{2}$ is the standard deviation of the noise vector $\mathbf{v}$.

In order to build a detectable strategy for nonzero taps, we use the structured LS algorithm which is expressed as following:

$$
\mathbf{r}=\operatorname{Sdiag}(\mathbf{b}) \mathbf{h}+\mathbf{n} \text {. }
$$

The nonzero values of the channel vector $\mathbf{h}$ are in the set of positions $\Theta=\left(z_{0}, z_{1}, \ldots, z_{D-1}\right)$. The total number of nonzero taps in the CIRs, $D$, is unknown a priori and generally $D<L<L_{c p}$ for sparse channels. $\mathbf{b}$ is a sparse vector, which indicates the sparse structure of the channels and its entries are given by

$$
b_{i}=\left\{\begin{array}{l}
1, \quad i \in \Theta \\
0, \text { otherwise } .
\end{array}\right.
$$

Similarly to the unstructured LS estimate (5) and (7), the structured LS estimate of sparse channels can be obtained once the estimate of the sparse vector $\mathbf{b}$ is acquired, i.e.,

$$
\hat{\mathbf{h}}=[\operatorname{Sdiag}(\hat{\mathbf{b}})]^{+} \mathbf{r}=\operatorname{diag}(\hat{\mathbf{b}}) \mathbf{h}+\mathbf{v} .
$$

where $\hat{\mathbf{b}}$ is the estimate of $\mathbf{b}$. Due to the sparseness of $\hat{\mathbf{b}}$, the matrix $\operatorname{Sdiag}(\hat{\mathbf{b}})$ is not fullrank and hence the pseudo-inverse $[\cdot]^{+}$is applied in the above equation.

Obviously seen from (11), the estimated results of CIRs are fully contaminated by noise. Therefore, we essentially have a binary hypothesis test for the channel tap:

$$
\left\{\begin{array}{l}
H_{0 i}: h_{i} \cdot 0+\left.v_{i}\right|_{b_{i}=0}=v_{i} \\
H_{1 i}: h_{i} \cdot 1+\left.v_{i}\right|_{b_{i}=1}=h_{i}+v_{i}
\end{array}\right.
$$

where $H_{0 i}$ indicates that $h_{i}$ is a zero tap in the $i$ th tap position whereas $H_{1 i}$ indicates that $h_{i}$ is a nonzero tap in the $i$ th tap position. Note that the above hypothesis test is independent of the number of nonzero taps, which render the consequent detection of channel taps.

Based on the above analysis, it is feasible to determine the nonzero taps out of noise by an appropriate threshold. To achieve this purpose, the universal threshold, proposed in [2] for compressed channel sensing can be employed:

$$
\lambda=\sqrt{4 \sigma_{v}^{2} \log L_{c p}} \text {. }
$$

The paper [17] also concluded that compressed channel sensing techniques cannot essentially be improved in the sense of mean-squared error in terms of the universal threshold in [2]. From (11), an accurate standard deviation $\sigma_{v}^{2}$ of each element in the noise vector $\mathbf{v}$, is necessary to the appropriate threshold. However, it is difficult to obtain an effective estimate of standard deviation of each element in $\mathbf{v}$ when the noise vector $\mathbf{v}$ and the channel vector $\mathbf{h}$ are combined together.

In the following part, an efficient threshold is proposed based on the estimated CIRs and the statistics of noise. The square of the amplitude of complex white Gaussian noise $v_{i}$ in $i$ th element of vector $v$ follows Exponential distribution [18] [19]. The cumulative distribution function of Exponential distribution is expressed as:

$$
F(x)=1-e^{\left(\frac{-x}{\sigma_{v}^{2}}\right)} .
$$

If $F(x)=0.5$, the value of corresponding $x$ is the median of $\left|v_{i}\right|^{2}$. In terms of the median value of $\left|v_{i}\right|^{2}$, the standard deviation of $v_{i}$ can be expressed as [19]:

$$
\sigma_{v}^{2}=\operatorname{median}\left(\left|v_{i}\right|^{2}\right) / \log 2 \text {. }
$$


If the nonzero channel taps in $\hat{\mathbf{h}}$ are removed from (11) and (12), the remaining zerovalue taps is regarded as noise, which can be applied to obtain a good estimate of the noise standard deviation $\sigma_{v}^{2}$. Therefore, the estimate noise standard deviation $\hat{\sigma}_{v}^{2}$ :

$$
\hat{\sigma}_{v}^{2}=\operatorname{median}\left(|\hat{\mathbf{h}}-\operatorname{diag}(\hat{\mathbf{b}}) \hat{\mathbf{h}}|^{2}\right) / \log 2 \text {. }
$$

Using this improved noise standard deviation $\hat{\sigma}_{v}^{2}$, an effective threshold $\Gamma$ can be obtained as

$$
\Gamma=\sqrt{4 \hat{\sigma}_{v}^{2} \log \left(L_{c p}-\hat{D}\right)}
$$

where $\hat{D}=\operatorname{sum}\left(\hat{b}_{i}\right)$ is the estimate of the total number of nonzero taps. After deleting the zero taps via the above threshold, the channel estimation efforts focus on a smaller number of nonzero taps by using the structured LS estimator, thus producing more accurate estimates of channel coefficients. In this scheme, only the coarse knowledge of channel length $L$ is required, but no knowledge of $D$.

The main steps of the proposed channel estimation algorithm is summarized as

Step 1: Initialize the channel estimation $\hat{\mathbf{h}}_{0}=\hat{\mathbf{h}}_{L S}$ by the unstructured LS in (5) and $\hat{\mathbf{b}}_{0}=0$.

Step 2: For-loop: For $j=1,2, \ldots, J_{\max }$

(2-1) Determine the estimated noise standard deviation by:

$$
\hat{\sigma}_{v}^{2}=\operatorname{median}\left(\left|\hat{\mathbf{h}}_{j-1}-\operatorname{diag}\left(\hat{\mathbf{b}}_{j-1}\right) \hat{\mathbf{h}}_{j-1}\right|^{2}\right) / \log 2 .
$$

(2-2) Determine the threshold by $\Gamma=\sqrt{4 \hat{\sigma}_{v}^{2} \log \left(L_{c p}-\hat{D}_{j-1}\right)}$.

(2-3) Make the hard decision for nonzero channel taps by

$$
\hat{b}_{j}=\frac{\operatorname{sign}\left(\left|\hat{h}_{j-1}(l)\right|-\Gamma\right)+1}{2}, \quad\left(l=0,1, \ldots, L_{c p}-1\right) .
$$

and the total number of nonzero taps is determined by $\hat{D}_{j}=\operatorname{sum}\left(\hat{b}_{j}\right)$.

(2-4) Calculate the structured estimate $\hat{\mathbf{h}}_{j}=\left[\operatorname{Sdiag}\left(\hat{\mathbf{b}}_{j}\right)\right]^{+} \mathbf{r}$ by the structured LS in (11).

If $\left\|\hat{\mathbf{h}}_{j}-\hat{\mathbf{h}}_{j-1}\right\|^{2}>\varepsilon$ or $j<J_{\max }$, increase $j$ and return to (2-1). Otherwise, the current estimate $\hat{\mathbf{h}}_{j}$ is the solution of CIR estimation. In our simulations, the number of iteration is typically limited to $J_{\max }=3$.

\section{Simulation Results and Analysis}

A 16QAM modulated OFDM system we consider in simulations has $N=512$ subcarriers and $N_{p}=128$ pilot subcarriers. $L_{c p}$ is set to 64 symbols. The length of channel impulse responses is $L=40$. The channel estimation algorithms are compared using the following channel models, which are illustrated in Figure 1: (a) 3-tap sparse channel in [20]; (b) 6tap sparse channel in [12] with the power delay profile $p(l)=e^{-0.125 l}$. The type of channel model (b) is also widely used in many literatures. Meanwhile, the channels are assumed to be time-invariant over the OFDM symbol duration. 

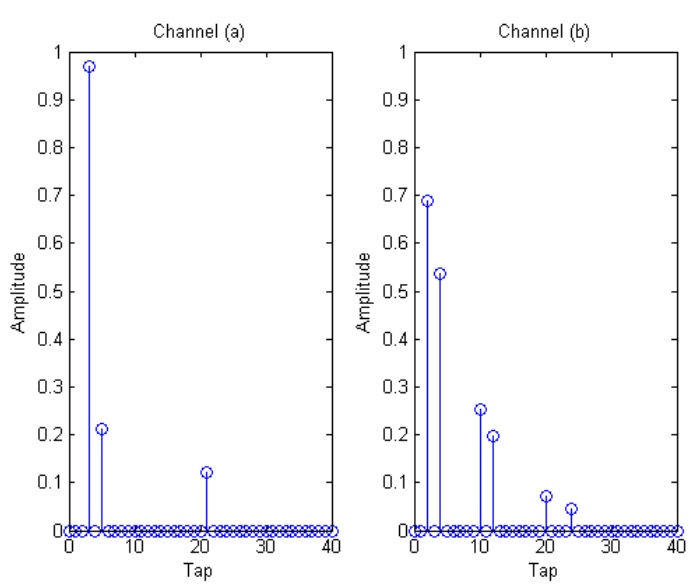

Figure 1. The Impulse Response of Channel (a) and Channel (b)

The performance of different channel estimators is evaluated in terms of the MSE, defined as $\operatorname{MSE}(\mathrm{dB})=10 \log _{10}\left(1 / N_{m} \sum_{n=1}^{N_{m}}\|\hat{\mathbf{h}}-\mathbf{h}\|^{2}\right)$ where $N_{m}$ is the total number of Monte Carlo iterations. In Figure 2 and Figure 3, the SNR variation ranges from 5 to $35 \mathrm{~dB}$ and the MSE of all the estimation algorithms are tested under two different channel conditions and averaged over 2000 Monte Carlo iterations for each SNR. The MSE of OMP [9], MIDE [14] and TMSE [15] are also plotted for comparison purposes. In addition, we plot the corresponding Cramer-Rao Bound (CRB) for unstructured channel estimation CRB-U $=\operatorname{trace}\left(\mathbf{S}^{T} \mathbf{S}\right)^{-1}$ and structured channel estimation CRB-S $=\operatorname{trace}\left(\mathbf{S}_{b}{ }^{T} \mathbf{S}_{b}\right)^{+}$, where $\mathbf{S}_{b}=\operatorname{Sdiag}(\mathbf{b})$. Here, the structured channel estimation CRB is obtained by using the exact knowledge of channel taps.

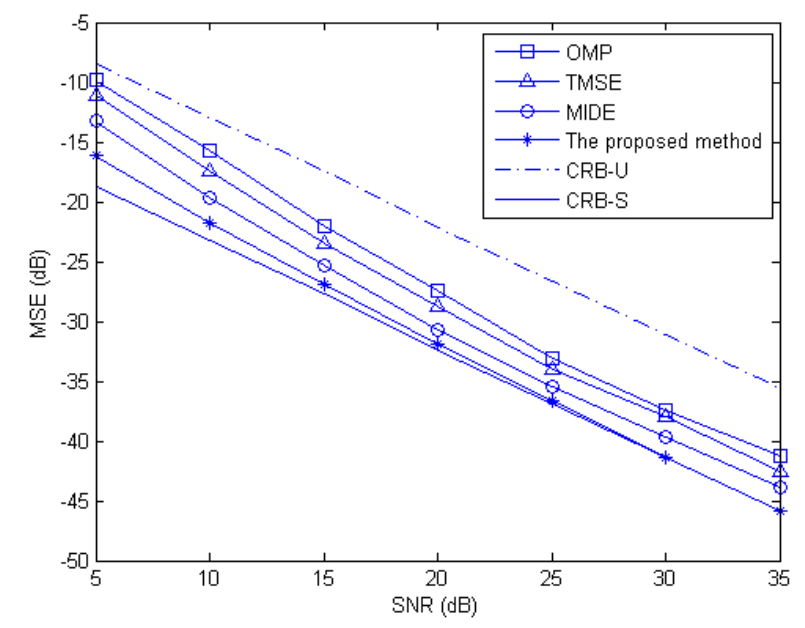

Figure 2. MSE Performance of Channel (a)

From Figure 2 and Figure 3, the proposed method has the best performance among all the estimators. Since it applies the statistical relationship of noise and the sparse CIRs to obtain a more precise threshold, the accuracies of the proposed method are closer to CRB_S than those of other methods. The OMP algorithm results in significant performance degradation as the number of nonzero taps increases in that it is very hard to choose a good stopping rule[14]. 


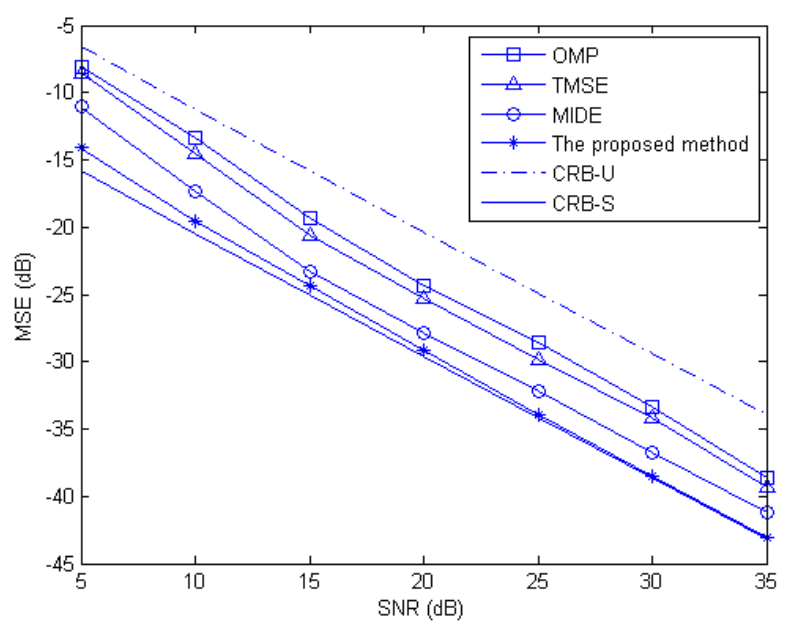

Figure 3. MSE Performance of Channel (b)

In order to further test the performance of the proposed estimation method, Figure 4 and Figure 5 show the average probability of correct detection of the nonzero taps by different estimation methods under different channel conditions. The simulation is repeated over 100 times. From Figure 4 and Figure 5, it is shown that the proposed estimation method has more accurate detection capabilities of channel taps in comparison with the existing methods. According to the simulation results, we conclude that the proposed method is effective in detecting the position of the nonzero taps. TMSE are very sensitive to the errors of tap missing and false detection. Once a false happens, the accuracy of TMSE will decrease. MIDE also include a threshold for channel tap detection, but it did not provide any mathematical analysis or simulation result on the selection of this threshold and hence it cannot guarantee the accuracy under any sparse channels. To determine the effect of $J_{\max }$ on the proposed method, the MSE for different channels is plotted in Figure 6 with $J_{\max }$ ranging from 1 to 8 . It is shown that the MSE becomes stable when $J_{\max } \geq 3$. It is the reason that we fix $J_{\max }$ to be 3 in our estimation algorithm.

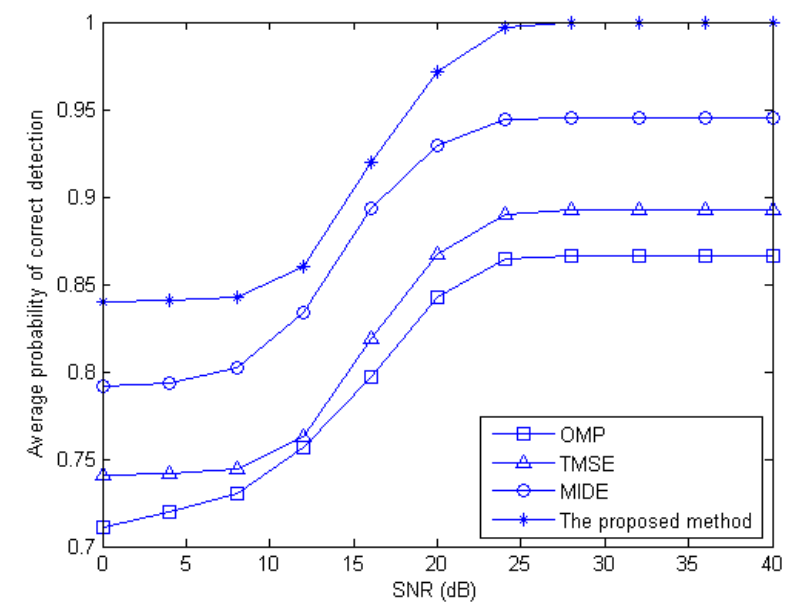

Figure 4. Average Probability of Correct Detection of Channel (a) 


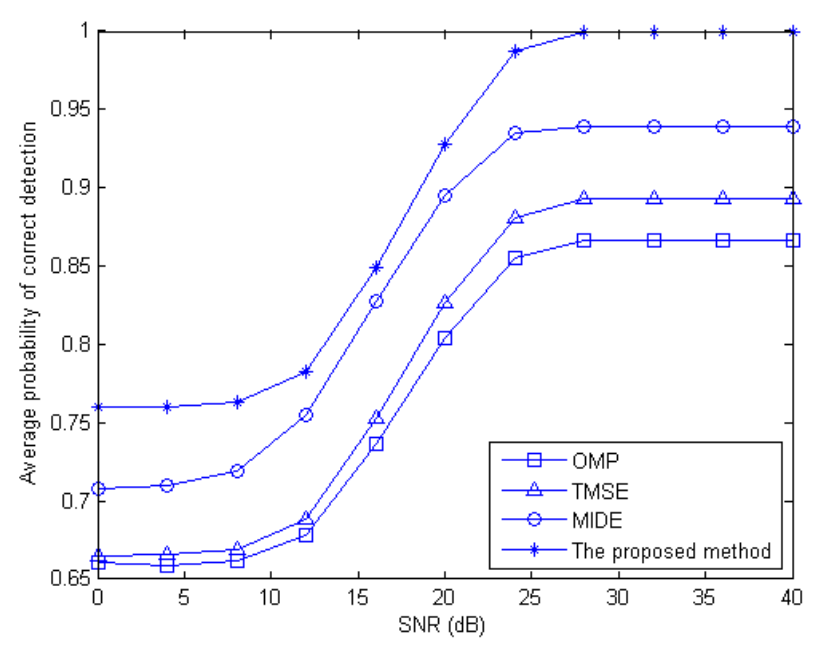

Figure 5. Average Probability of Correct Detection of Channel (b)
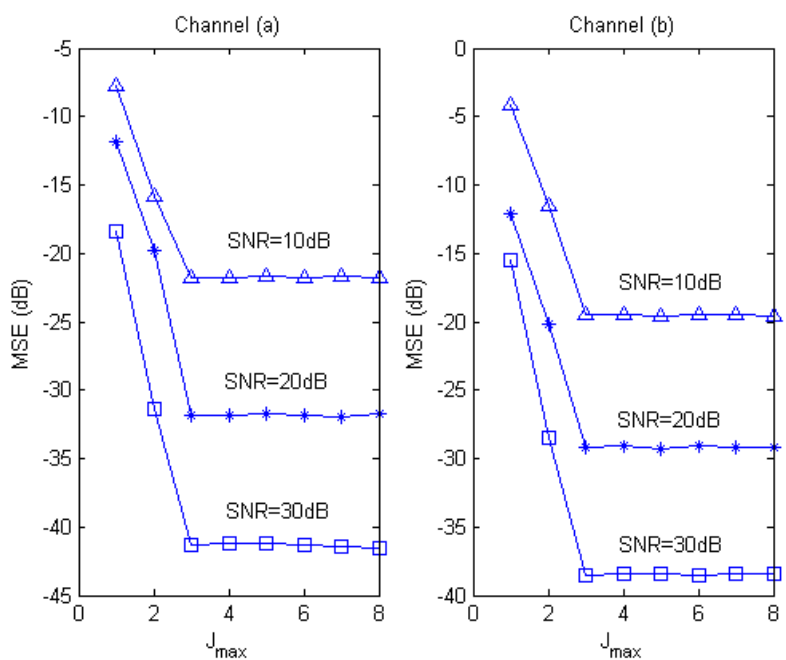

Figure 6. The Effect of $J_{\max }$ on MSE Performance of the Proposed Method

The complexities of these algorithms in terms of the CPU running times are compared in Table 1, where SNR is set to be $35 \mathrm{~dB}$. The experiments are performed under the aforementioned two channels using MATLAB on the computer with a CPU of Intel Core i5 and 2.0GB RAM. In Table 1, all the estimators have the similar results under different channel conditions. It is also shown that the proposed method is faster than OMP, MIDE and TMSE, and its running times are close to those of TMSE. Since OMP cannot always guarantee successful sparse recovery and sometimes it runs out of all matrix columns, the time cost of OMP is the highest among these channel estimators.

Table 1. Average CPU Running Times of different Channel Estimators

\begin{tabular}{ccc}
\hline \multirow{2}{*}{ Channel estimators } & \multicolumn{2}{c}{ CPU running times (in Seconds) } \\
\cline { 2 - 3 } & Channel (a) & Channel (b) \\
\hline OMP & 33.618 & 33.852 \\
TMSE & 20.063 & 20.281 \\
MIDE & 26.229 & 26.397 \\
Proposed method & 18.529 & 18.781 \\
\hline
\end{tabular}




\section{Conclusion}

Channel estimation methods with good estimation performance and moderate computational complexity would significantly benefit wireless communication systems. In this paper, we have investigated the possible improvement of sparse channel estimations by proposing a new sparse channel estimation method. In view of the characteristics of the sparse channel, a novel threshold-based channel estimation method is developed based on the estimated noise standard derivation and structured LS estimation method. This threshold scheme of the proposed method leads to superior channel estimation performance by achieving a good trade-off for nulling out more channel taps corresponding to noise. The simulation results show that compared with the existing methods, the proposed method exhibits not only better performance in terms of MSE and average probability of correct detection of nonzero channel taps but also better computational complexity in average CPU running times. Moreover, in contrast to the OMP method, our method does not require a priori information about the number of nonzero channel taps.

\section{Acknowledgements}

This work is supported by the National Natural Science Foundation of China (11274253), the Scientific Research Program Funded by Shaanxi Provincial Education Department (14JK1673) and the Program of International Cooperation and Exchanges in Science and Technology Funded by Shaanxi Province (2016KW-033).

\section{References}

[1] Goldsmith, "Wireless Communications", New York: Cambridge Univ. Press, (2005).

[2] W. U. Bajwa, J. Haupt, A. M. Sayeed, R. Nowak, "Compressed Channel Sensing: A New Approach to Estimating Sparse Multipath Channels", Proceedings IEEE, vol. 98, (2010).

[3] R. J. M. Cramer, R. A. Scholtz, and M. Z. Win, "Evaluation of an ultra-wide-band propagation channel", IEEE Trans. Antennas Propag., vol. 50, (2002).

[4] A. F. Molisch, "Ultrawideband propagation channels-Theory, measurement, and modeling", IEEE Trans. Veh. Technol., vol. 54, (2005).

[5] Z. Yan, M. Herdin, A. M. Sayeed, and E. Bonek, "Experimental study of MIMO channel statistics and capacity via the virtual channel representation”, Univ. Wisconsin-Madison, Madison, Tech. Rep., (2007).

[6] L. Vuokko, V. M. Kolmonen, J. Salo, and P. Vainikainen, "Measurement of large-scale cluster power characteristics for geometric channel models", IEEE Trans. Antennas Propag., vol. 55, (2007).

[7] S. F. Cotter, B. D. Rao, "Matching pursuit based decision-feedback equalizers", IEEE Int. Conf. Acoust. Speech Signal Process., vol. 1, (2000), pp. 2713-2716.

[8] S. F. Cotter, B. D. Rao, "Sparse channel estimation via matching pursuit with application to equalization", IEEE Trans. Commun., vol. 50, (2002).

[9] G. Z. Karabulut, A. Yongacoglu, "Sparse channel estimation using orthogonal matching pursuit algorithm", IEEE Vehicular Technology Conference, (2004), pp. 3880-3884.

[10] C. J. Wu, D. W. Lin, "A group matching pursuit algorithm for sparse channel estimation for OFDM transmission”, IEEE Int. Conf. Acoust. Speech Signal Process., (2006), pp. 429-432.

[11] W. Li, J. C. Preisig, "Estimation of rapidly time-varying sparse channels", IEEE J. Ocean. Eng., vol. 32, (2007).

[12] M. R. Raghavendra, K. Giridhar, "Improving channel estimation in OFDM systems for sparse multipath channels", IEEE Signal Process. Lett., vol. 12, (2005).

[13] C. Carbonelli, U. Mitra, "A simple sparse channel estimator for underwater acoustic channels", MTS/IEEE Oceans, (2007), pp. 1-6.

[14] F. Wan, U. Mitra, A. Molisch, "The modified iterative detector/estimator algorithm for sparse channel estimation," MTS/IEEE Oceans, (2010), pp. 1-6.

[15] Z. Jellali, L. N. Atallah, "Threshold-based channel estimation for MSE optimization in OFDM systems", EURASIP, (2012).

[16] G. Caire, U. Mitra, "Structured multiuser channel estimation for block-synchronous DS/CDMA", IEEE Transactions on Communications, vol. 49, (2001).

[17] E. Candes, M. Davenport, "How well can we estimate a sparse vector", Applied and Computational Harmonic Analysis, vol. 34, (2013).

[18] Bernard Picinbono, "Second-order complex random vectors and normal distributions", IEEE Transactions on Signal Processing, vol. 44, no. 10, (1996). 
[19] A. Papoulis, S. Pillai, Probability, Random Variables and Stochastic Processe., McGraw Hill Higher Education, (2001).

[20] A. Maltsec, "Channel models for $60 \mathrm{GHz}$ WLAN systems", IEEE doc.802.11-09/0334r8, (2010).

\section{Authors}

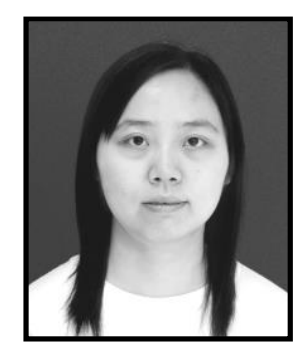

Xiaolin Shi, she received her M. Sc. and M. Phil. degrees from Northwestern Polytechnical University in 2005 and from City University of Hong Kong in 2006, respectively. She currently works as an associate professor of School of Electronics Engineering at Xi'an University of Posts \& Telecommunications, China. Her research interests include wireless communications and statistical signal processing.

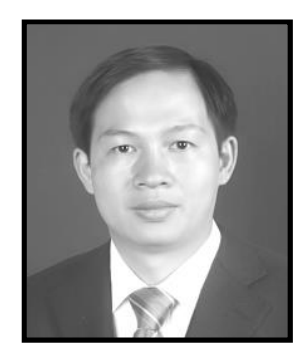

Yixin Yang, he received his Ph.D. degree from Northwestern Polytechnical University in 2002. He had been a Post-doctoral researcher from 2002 to 2004 at Nanyang Technological University, Singapore. Since June 2004, he has been working as a professor of School of Marine Science and Technology at Northwestern Polytechnical University, China. His research interests include underwater acoustics, acoustic signal processing, and acoustic system design.

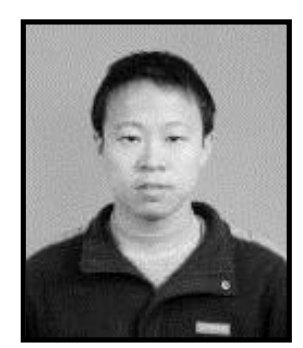

Long Yang, he was born in 1988, Wuhu, Anhui Province. He is currently pursuing his Ph.D. degree at Northwestern Polytechnical University. His research interests are array signal processing and acoustic signal detection. 